\title{
Calving Front Location Extraction of Antarctic Peninsula from TanDEM-X DEM Products
}

\author{
Yuting Dong 1, 2, Lukas Krieger ${ }^{2}$, Dana Floricioiu ${ }^{2}$, Ji Zhao ${ }^{1,3}$ \\ ${ }^{1}$ China University of Geosciences \\ ${ }^{2}$ Remote Sensing Technology Institute (IMF), German Aerospace Center (DLR), Oberpfaffenhofen, Germany \\ ${ }^{3}$ German Remote Sensing Data Center (DFD), German Aerospace Center (DLR), Oberpfaffenhofen, Germany
}

\begin{abstract}
I Abstract
As one important parameter of ice dynamics, the calving front location (CFL) of outlet glaciers and ice-shelf reveals the dynamic response of the glaciers or ice shelves to the changing ocean circulation or warming climate as well as the structural conditions.

It is very challenging to automatically extract CFLs from the optical and SAR remote sensing images especially along complex coastlines like on Antarctic Peninsula (AP). The major difficulty lies in distinguishing different surface types with similar optical spectral or radar backscattering characteristics. Our proposed method is an attempt to replace the manual delineation of CFL still used to derive such geophysical products e.g for the Antarctic Digital Database (ADD) coastline.
\end{abstract}

Our research work includes the following:

- As data source for automatic CFL extraction we used high-resolution DEM;

- We generate a novel feature image based on DEM roughness and elevation;

- The path propagation method is applied to the feature image for automatic CFL extraction;

- Validation of the extracted CFL with the ADD coastline.

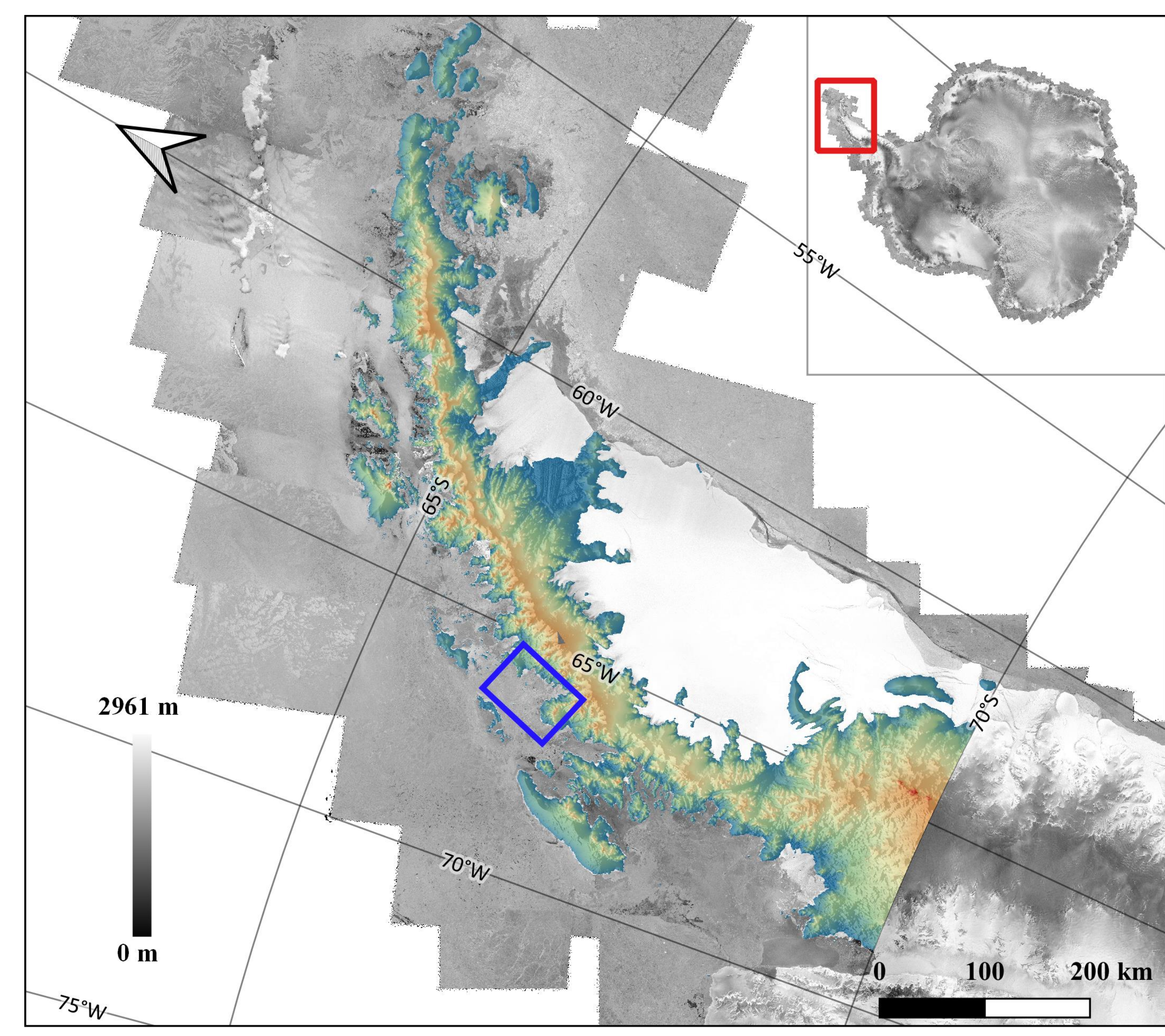

Figure 1 DEM hillshade map of Antarctic Peninsula (AP). The blue rectangle marks the experimental area used in this poster.

The TanDEM-X global DEM with $12 \mathrm{~m}$ posting is used as experimental data in the Darbel Bay located on the western coast of AP (Figure 1). In the future the proposed method will be tested on the whole AP coast with the $12 \mathrm{~m}$ TanDEM-X global DEM as well as with time series of TanDEM-X DEMs.

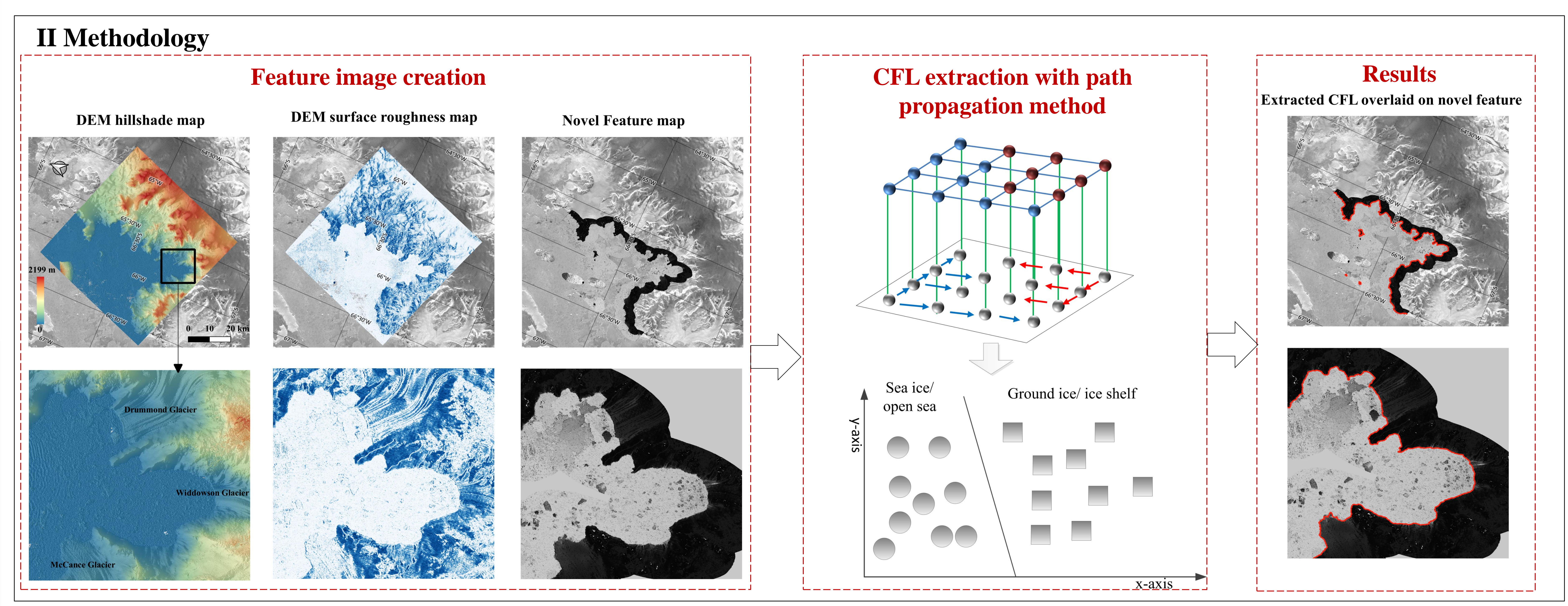

III Experimental results
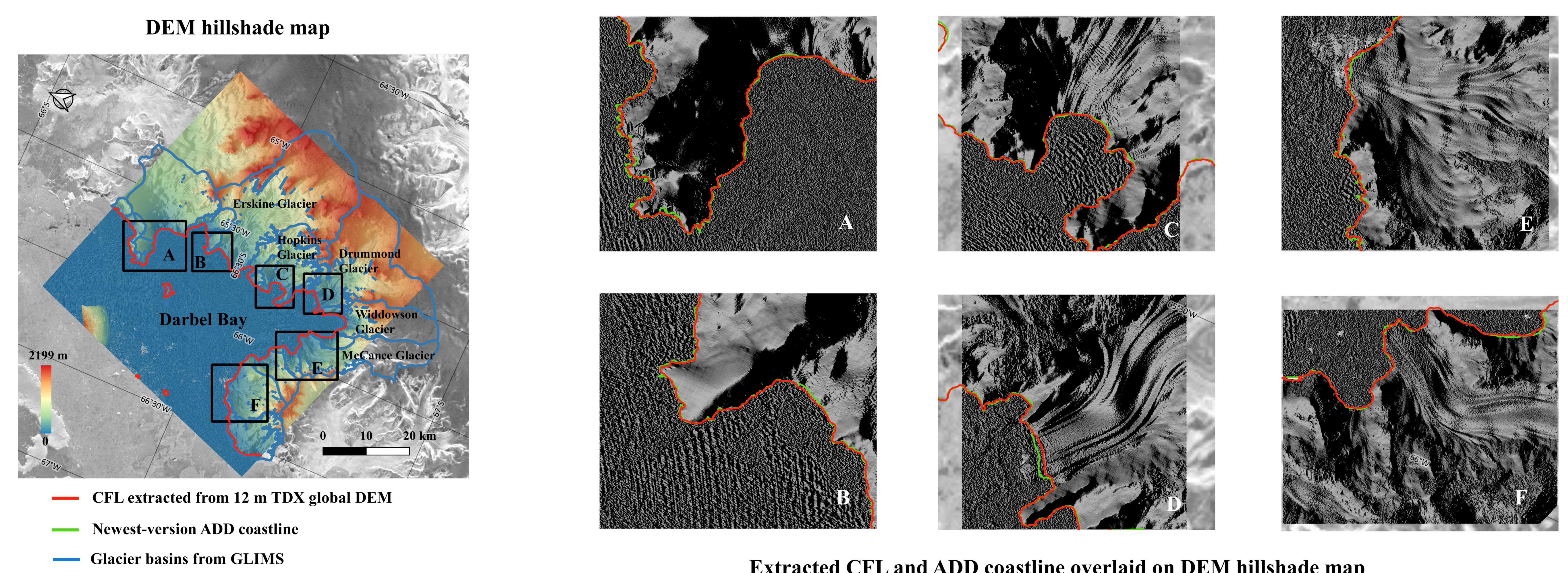

Extracted CFL and ADD coastline overlaid on DEM hillshade map 